\title{
CLINICAL IMPLICATIONS OF BASIC RESEARCH
}

\section{Sorting Out Cholesterol and Coronary Artery Disease}

Patrick Linsel-Nitschke, M.D., Nilesh J. Samani, M.D., M.B., Ch.B., and Heribert Schunkert, M.D.

A genomewide association study published in the Journal 3 years ago reported the identification of

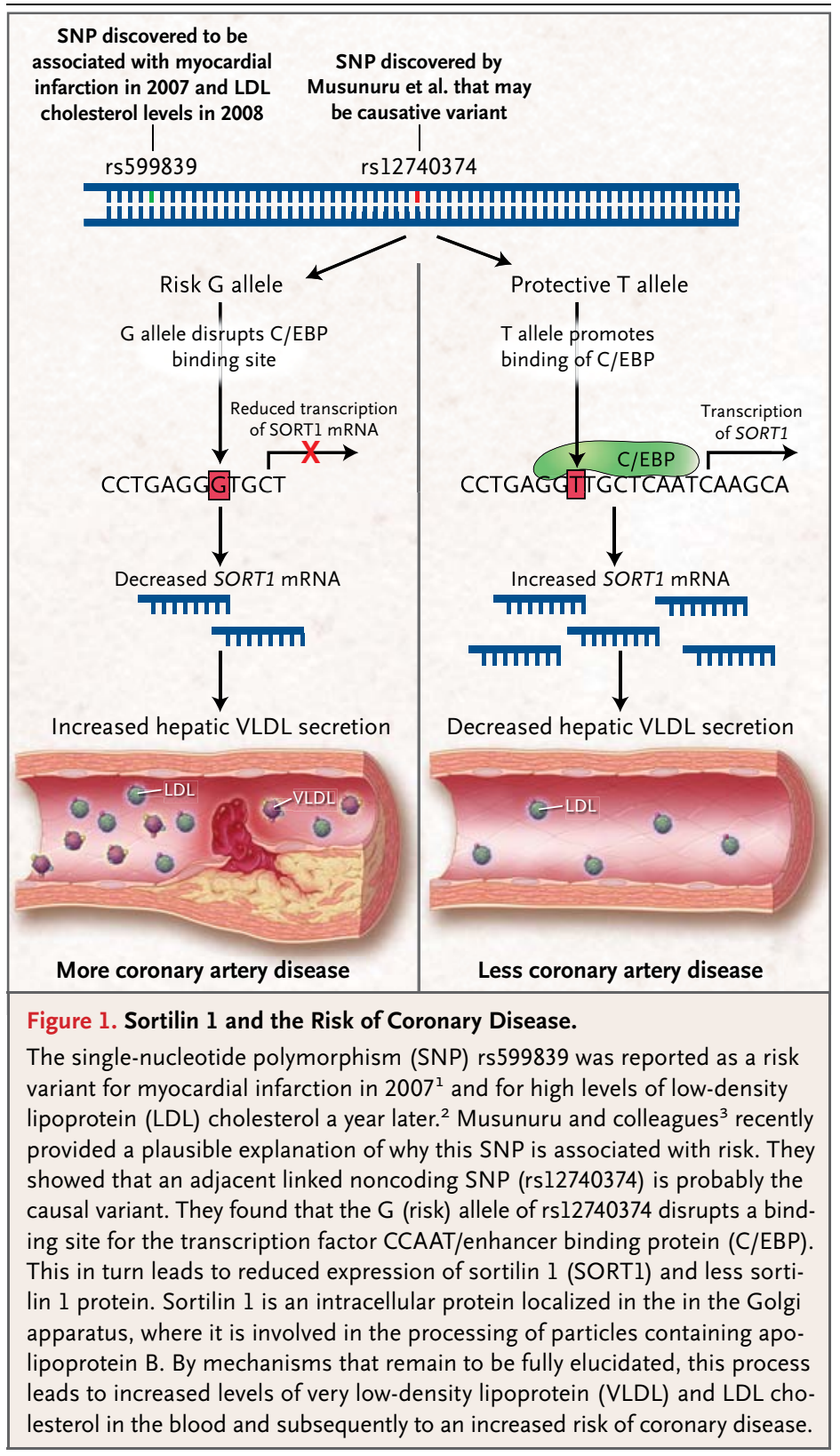

a DNA variant on chromosome $1 \mathrm{p} 13$ that increased the relative risk of coronary artery disease by $29 \%$ per allele. ${ }^{1} \mathrm{~A}$ few months later, the same genetic variant was also found - again through a genomewide association approach - to increase serum levels of low-density lipoprotein (LDL) cholesterol. ${ }^{2}$ At that time, little was known about the genes at the $1 \mathrm{p} 13$ locus, so the functional link between the DNA variant and the increase in LDL cholesterol and coronary risk was puzzling. Basic research carried out by Musunuru et al. ${ }^{3}$ now sheds light on the underlying pathway and identifies a novel target for prevention and treatment of atherosclerosis.

Using the previously identified risk allele as an anchor, Musunuru et al. first explored whether there was a relationship between the risk variant and the expression of any of the genes on chromosome 1p13. This approach was based on the hypothesis that the risk variant might alter the abundance of a protein that is critical to the pathogenic mechanism (i.e., a bridgehead between risk allele and disease phenotype). Indeed, the investigators showed that specifically in the liver, an organ intimately involved in lipid metabolism, three genes at $1 \mathrm{p} 13$ were expressed at reduced levels in carriers of the risk allele.

Modulation of multiple genes by the risk allele suggested involvement of a regulatory element. In order to find this element, the investigators sequenced the $1 \mathrm{p} 13$ region. They found additional DNA variants and subsequently tested these variants for association with LDL cholesterol, an approach called fine mapping. They thereby narrowed down the region containing the probable causal variant to a small segment. Bioinformatic analysis indicated that one of the variants (rs12740374) within this noncoding segment lies within a putative binding site for CCAAT/enhancer binding protein (C/EBP), a transcription factor; the risk allele is predicted to disrupt binding. It was thus tempting to speculate that this partic- 
ular single-nucleotide polymorphism (SNP) is directly involved in the regulation of gene expression at the chromosome $1 \mathrm{p} 13$ locus. Indeed, the investigators confirmed physical binding of C/EBP to a small stretch of DNA containing the protective variant but not the risk variant of rs12740374. They then observed increased expression of a reporter gene in liver cells transfected with a vector carrying the intact but not the variant $\mathrm{C} / \mathrm{EBP}$ binding site. The experiments suggest a chain of events linking specifically the risk variant of SNP rs12740374 to reduced expression levels of genes on chromosome 1p13 (Fig. 1).

The gene that was most affected in its expression was SORT1, encoding sortilin 1 protein. Consequently, Musunuru et al. tested the involvement of sortilin 1 in the metabolism of LDL cholesterol. Liver-specific overexpression of Sort1 in mice decreased serum levels of LDL cholesterol. ${ }^{3}$ Knockdown of sortilin 1 had the opposite effect, thereby mimicking the situation in humans who carry the risk variant of rs12740374. These data strongly suggest that SNP rs12740374 affects sortilin 1 expression and thus is responsible for the association between variants at chromosome $1 \mathrm{p} 13$ and LDL cholesterol and, by extension, the risk of coronary disease.

The next question was how increased expression of sortilin 1 functionally results in reduced serum levels of LDL cholesterol. Sortilin 1 is localized in the Golgi apparatus and endoplasmatic reticulum, where it appears to act as an intracellular sorting receptor for particles containing apolipoprotein B. ${ }^{4}$ Musunuru et al. found that hepatocytes in mice with sortilin 1 knockdown had a significant increase in the secretion of particles containing apolipoprotein $\mathrm{B}$, notably very-lowdensity lipoprotein (VLDL) cholesterol, whereas the overexpression of sortilin 1 was associated with decreased secretion of such particles (Fig. 1). Moreover, the overexpression of sortilin 1 was shown to enhance the endocytosis of LDL particles in vitro. ${ }^{5}$ Taken together, these findings provide strong experimental evidence for the involve- ment of sortilin 1 in the hepatic metabolism of lipoproteins containing apolipoprotein $\mathrm{B}$, although the precise mechanism awaits further elucidation. ${ }^{3-5}$

The occurrence of the risk variant of rs12740374 in multiple populations of different ancestry and its high prevalence $(68 \%$ in those of European ancestry) imply that this SNP affects lipoprotein levels around the globe through its modulatory effects on gene transcription. ${ }^{3}$ The resulting decrease of sortilin 1 expression appears to elevate levels of two atherogenic lipoproteins, VLDL and LDL, and thus acts as a double-edged sword with respect to atherosclerosis risk. The clinical importance of this pathway is defined by a substantial increase in the risk of coronary disease for every copy of the $1 \mathrm{p} 13$ risk allele. ${ }^{1,2,5}$ All told, the discovery of the C/EBP-sortilin 1 pathway provides an example of how genomewide association studies can initiate new biologic discoveries, leading to a better understanding of pathophysiology, which could ultimately translate into new approaches to treatment.

Disclosure forms provided by the authors are available with the full text of this article at NEJM.org.

From the Medizinische Klinik II, University of Lübeck, Lübeck, Germany (P.L.-N., H.S.); and the Department of Cardiovascular Sciences and Leicester National Institute for Health Research, Biomedical Research Unit in Cardiovascular Disease, University of Leicester, Leicester, United Kingdom (N.J.S.).

1. Samani NJ, Erdmann J, Hall AS, et al. Genomewide association analysis of coronary artery disease. N Engl J Med 2007; 357:443-53.

2. Kathiresan S, Melander O, Guiducci C, et al. Six new loci associated with blood low-density lipoprotein cholesterol, highdensity lipoprotein cholesterol or triglycerides in humans. Nat Genet 2008;40:189-97.

3. Musunuru K, Strong A, Frank-Kamenetsky M, et al. From noncoding variant to phenotype via SORT1 at the $1 \mathrm{p} 13$ cholesterol locus. Nature 2010;466:714-9.

4. Kjolby M, Andersen OM, Breiderhoff T, et al. Sort1, encoded by the cardiovascular risk locus $1 \mathrm{p} 13.3$, is a regulator of hepatic lipoprotein export. Cell Metab 2010;12:213-23.

5. Linsel-Nitschke P, Heeren J, Aherrahrou Z, et al. Genetic variation at chromosome $1 \mathrm{p} 13.3$ affects sortilin mRNA expression, cellular LDL-uptake and serum LDL levels which translates to the risk of coronary artery disease. Atherosclerosis 2010; 208:183-9.

Copyright $(2010$ Massachusetts Medical Society. 